\title{
Blockage of Peripheral NPY Y1 and Y2 Receptors Modulates Baroreflex Sensitivity of Diabetic Rats
}

\author{
Jing Liu ${ }^{a}$ Ning Wang ${ }^{a}$ Fang Xie Li-Hua Sun Qing-Xin Chen Jian-Han Ye Ben-Zhi Cai \\ Bao-Feng Yang Jing Ai
}

Department of Pharmacology, Harbin Medical University (the State-Province Key Laboratories of Biomedicine-Pharmaceutics of China), Harbin, Heilongjiang Province, China; ${ }^{a}$ Contributed equally to this paper

\section{Key Words}

Neuropeptide Y $\bullet$ NPY Y1 receptor $•$ NPY Y2 receptor $•$ Diabetic rats $•$ Baroreceptor reflex sensitivity $\bullet$ Cardiac function

\begin{abstract}
Background/Aims: Abnormal baroreceptor reflex sensitivity (BRS) and elevated plasma neuropeptide $Y$ (NPY) are prevalent in diabetic patients. The present study was conducted to determine whether NPY Y1 receptor (Y1R) and NPY Y2 receptor (Y2R) contribute to the regulatin of BRS in diabetic rats. Methods: Diabetes mellitus (DM) rats with hyperlipidemia were developed by an emulsion diet enriched with fat, sucrose and fructose followed by streptozocin (STZ). Y1R and Y2R specific antagonists (BIBP 3226 and BIIE 0246) were administered by a mini-osmotic pump. Systolic blood pressure (SBP), heart rate (HR), BRS and heart functions, as well as the plasma NPY and lipid level were measured after treatment for 4 weeks. Results: Both BIBP 3226 and BIIE 0246 treatment reversed the elevated total cholesterol (TC) and low density lipoprotein (LDL-C) level, and reduced high density lipoprotein (HDL-C) level in DM rats. BIIE 0246 may attenuate the increased triglyceride (TG) level in DM rats. In addition, neither BIBP 3226 nor BIIE 0246 treatment produced significant effects on BRS, SBP or HR $(P>0.05)$ in DM rats, even after PE and SNP challenge. However, BIBP 3226 and BIIE 0246 further impaired LVSP, LVEDP, $+\mathrm{dp} / \mathrm{dt}_{\max }$ and $-\mathrm{dp} / \mathrm{dt}_{\max }$. Conclusion: This study provided us with the evidence that the inhibition of peripheral Y1R and Y2R did not affect impaired BRS but amplified the deterioration of the compromised cardiac function in STZ-induced DM rats with hyperlipidemia.
\end{abstract}




\section{Introduction}

The global prevalence of Type 2 diabetes mellitus (T2DM) has risen dramatically over the past two decades. In diabetic patients, cardiovascular complications are responsible for the high rate of morbidity and mortality [1]. It is well documented that diabetes mellitus may increase the risk for myocardial infarction, atherosclerosis, stroke and limb loss. As another serious complication of diabetes, cardiovascular autonomic neuropathy (CAN) can cause dysfunctional heart-rate control and abnormal vascular dynamics because of the damages to the autonomic nerve fibers that govern the heart and blood vessels $[2,3]$. Recently, an epidemiological study also reported that the prevalence of CAN to be at $34 \%$ in 524 patients with T2DM [4]. In diabetes mellitus, disrupted baroreceptor reflex control of heart rate provides the substrate for life-threatening arrhythmias and has been recognized as a risk factor for sudden cardiac death $[2,5,6]$. Previous studies documented that diabetes mellitus caused neural degeneration in nucleus ambiguous (NA), leading to the attenuation of HR control in OVE26 mice [7]. However, the mechanisms of abnormal BRS in diabetic mellitus are unclear and need to be explored.

As a sympathetic neurotransmitter, Neuropeptide Y (NPY) has attracted attentions as an important regulatory molecule in a variety of physiological and pathological processes such as food intake control [8] and cardiovascular regulation [9]. Increasing evidence suggest that the level of NPY in plasma is significantly elevated in hypertrophy [10], hypertension [11] and myocardial ischemia [12]. Elevated levels of plasma NPY have also been proposed as a prognostic marker for coronary diseases [13], heart failure [14] and myocardial ischemia [15]. Our previous studies demonstrated that long-term NPY administration led to abnormal BRS, and reversed chronic stress-induced baroreflex hypersensitivity in rats [16,17]. Clinical investigation has shown that the level of plasma NPY is positively correlated with the insulin level for patients with T2DM [18]. However, whether elevated NPY level is responsible for abnormal BRS in diabetes mellitus with hyperlipidemia remains unknown. Among the six identified NPY receptor subtypes, NPY Y1 receptor (Y1R) and NPY Y2 receptor (Y2R) are dominantly expressed and function in the cardiovascular system [19]. Consequently, the present study was designed to investigate the regulatory effect of peripheral Y1R and Y2R on BRS and heart function in diabetic rats with hyperlipidemia.

\section{Materials and Methods}

\section{Animals}

Male Wistar rats (230-270 g, 3-4 months old) were purchased from the Animal Center of the Second Affiliated Hospital of Harbin Medical University (Harbin, Heilongjiang Province, China) and housed under controlled temperature $\left(23 \pm 1^{\circ} \mathrm{C}\right)$ and humidity (55 $\left.\pm 5 \%\right)$, and a 12-h dark/light cycle. All the animals received food and water ad libitum. The experimental procedures were in agreement with the regulations of the Ethics Committees of Harbin Medical University (No. HMUIRB-2008-06).

Streptozotocin-induced diabetic models in rats

Diabetes was induced in Wistar rats in accordance with our previous study [20]. Briefly, fat emulsion was made up with lard (20\%), thyreostat (1\%), cholesterol (5\%), sucrose (5\%), fructose (5\%), sodium glutamate $(1 \%)$ and salt (6\%). The above ingredients were emulsified in $20 \%$ Tween 80 with $30 \%$ propylene glycol and stored at $4{ }^{\circ} \mathrm{C}$. Twenty-four rats were randomly divided into 4 groups ( 6 rats per groups). For the first 10 days, the diabetic model group, diabetic model treated with Y1R antagonist group (DM+antiY1R) and diabetic model treated with Y2R antagonist group (DM+antiY2R) were orally administrated with the prepared fat emulsion $(10 \mathrm{ml} / \mathrm{kg}$ ). These groups then received one daily dose of $40 \mathrm{mg} / \mathrm{kg}$ streptozocin (STZ, in $0.1 \mathrm{~mol} / \mathrm{L}$ citrate buffer solution, $\mathrm{pH} 4.2$, Sigma) via intraperitoneal (i.p.) injection once daily for 3 days. After STZ injection, the rats continued to be treated with fat emulsion until the 30 day time point. To confirm the establishment of DM, fasting blood glucose (FBG) levels were monitored after the last injection of STZ for $72 \mathrm{~h}$ with a Grace glucometer (Grace Medical, Inc. America), and a FBG $\geq 16.7 \mathrm{mmol} / \mathrm{l}$ or $300 \mathrm{mg} /$ 


\begin{tabular}{|c|c|c|}
\hline Cellular Physiolosy & Cell Physiol Biochem 2013;31:421-431 & \\
\hline and Biochemistry & $\begin{array}{l}\text { DOI: } 10.1159 / 000343379 \\
\text { Publisned onlne: March 12, } 2013\end{array}$ & $\begin{array}{l}\text { O } 2013 \text { S. Karger AG, Basel } \\
\text { www.karger.com/cpb }\end{array}$ \\
\hline
\end{tabular}

dl was considered to adequately model DM. The control group was orally treated with a $0.9 \%$ saline solution and i.p. injection of citrate buffer solution.

\section{Implantation of the ALZET mini-osmotic pump}

Y1R antagonist (BIBP 3226, Sigma-Aldrich, St. Louis, MO) and Y2R receptor antagonist (BIIE 0246, Tocris, Ellisville, MO, USA) were administrated to DM rats ranked as DM+antiY1R and DM+antiY2R group respectively for 4 weeks ( $85 \mu \mathrm{g}$ per $30 \mathrm{~d}$ ) via implanted ALZET mini-osmotic pumps (DURECT Corporation, USA). Rats in the control and DM groups were treated with equivalent quantities of PBS. The preparation of the mini-osmotic pump for implantion was performed according to the product brochures (DURECT Corporation, USA).

All rats were anesthetized with $40 \mathrm{mg} / \mathrm{kg}$ of sodium pentobarbital and a mini-osmotic pump containing a one-month supply of treatment (PBS, BIBP 3226, or BIIE 0246 as previously described) was subcutaneously implanted via a small incision in the skin between the scapulae of each rat. A minor pocket was made with a hemostat by spreading the subcutaneous connective tissues apart, the pump was inserted into the pocket while orienting the flow moderator to point to the opposite direction of the incision, and the incision was closed with wound sutures $[17,18]$

\section{Measurement of cardiac function}

After 4 weeks of treatment with PBS, BIBP 3226 or BIIE 0246, rats were anesthetized with sodium pentobarbital $(40 \mathrm{mg} / \mathrm{kg}$ ). Heart functions of all rats were measured according to our previous study [21]. Systolic blood pressure (SBP) and heart rate (HR) were monitored by a catheter that was inserted through the right common carotid artery and into the left ventricle. Indicators of the systolic function of the heart such as left ventricular systolic pressure (LVSP) and the maximum rate of change in left ventricular pressure during the isovolumic contraction period $\left(+\mathrm{d} p / \mathrm{d} t_{\max }\right)$ were studied using a BL-420 Data Acquisition \& Analysis System (Chengdu Tme Technology Co., Ltd, China). To assess the diastolic function of heart, left ventricular diastolic pressure (LVEDP) and the maximum rate of change in left ventricular pressure during the isovolumic relaxation period $\left(-\mathrm{d} p / \mathrm{d} t_{\max }\right)$ were recorded.

\section{Surgical procedures}

As described in previous studies [22-24], rats were anesthetized with sodium pentobarbital (40 mg/ $\mathrm{kg}$ ) by i.p. injection. The eye blinking and withdrawal reflex were employed as indices for evaluation of the anesthetic condition. Supplemental doses of anesthetics ( $0.1 \mathrm{ml}$ of $1 \%$ sodium pentobarbital) were given every $30 \mathrm{~min}$ to maintain the anesthetic state. The left femoral artery and right femoral vein were exposed and tapered polyethylene catheters $(0.5 \mathrm{~mm}$ diameter-tip), pretreated with heparinized saline, were inserted respectively. Arterial blood pressure (ABP) was recorded in the left femoral artery and vasoactive drugs were administered through right femoral vein.

\section{Baroreflex sensitivity study}

The blood pressure catheter was connected to a blood pressure transducer (MIT0699; AD Instruments, Australia) and placed in a horizontal position level with the heart. Baseline mean arterial blood pressure (MABP), HR and serial responses to the vasoactive stimulations were studied. ABP was recorded automatically and HR was studied by pulse pressure with the rate-meter function of the BL-420 Data Acquisition \& Analysis System. Phenylephrine (PE) and sodium nitroprusside (SNP) were freshly diluted with $0.9 \% \mathrm{NaCl}$ solution and delivered at the following dosages: 16, 32, 64, 128, and $256 \mu \mathrm{g} / \mathrm{ml}$ for PE; 10 , $20,40,80$, and $160 \mu \mathrm{g} / \mathrm{ml}$ for SNP. The rate of injection was kept at $0.04 \mathrm{ml} / 100 \mathrm{mg}$. Baseline MABP and HR were monitored for $30 \mathrm{~s}$ before the first drug administration. The changes of MABP and HR were recorded and averaged every second, and the exogenous stimulation was not given until the ABP and HR reached a plateau. At each dose of PE or SNP, the MABP changes over the baseline ABP level ( $\triangle M A B P$ ) and the maximal $\mathrm{HR}$ responses relative to the baseline HR level $(\triangle \mathrm{HR})$ were recorded. To evaluate the BRS elicited by each dose of PE or SNP, the mean ratio of the HR change over the MABP change $(\triangle \mathrm{HR} / \triangle \mathrm{MABP})$ was calculated. Respective dose-dependent curves for $\triangle \mathrm{MABP}$ and $\triangle \mathrm{HR} / \triangle \mathrm{MABP}$ were also depicted for each group. The $\triangle$ HR- $\triangle$ MABP curves were plotted to show the maximal HR responses induced by altered MABP. All curves were fitted by Boltzmann equation with GraphPad Prism 5.0 software (GraphPad Software Inc., San Diego, CA, USA). 


\begin{tabular}{cllll}
\hline Groups & TC $(\mathrm{mmol} / \mathrm{l})$ & $\mathrm{TG}(\mathrm{mmol} / \mathrm{l})$ & HDL-C $(\mathrm{mmol} / \mathrm{l})$ & LDL-C $(\mathrm{mmol} / \mathrm{l})$ \\
\hline Ctl & $1.24 \pm 0.09$ & $0.57 \pm 0.04$ & $0.57 \pm 0.06$ & $0.18 \pm 0.01$ \\
DM & $2.07 \pm 0.16^{* *}$ & $0.89 \pm 0.08^{*}$ & $0.67 \pm 0.07$ & $0.63 \pm 0.05^{* *}$ \\
DM+antiY1R & $1.36 \pm 0.08^{+}$ & $0.78 \pm 0.07^{*}$ & $029 \pm 0.06^{* *_{+}}$ & $0.42 \pm 0.04^{* *+}$ \\
DM+antiY2R & $1.15 \pm 0.05^{++}$ & $0.44 \pm 0.06^{*+}$ & $0.34 \pm 0.04^{*+}$ & $0.41 \pm 0.03^{* *+}$ \\
\hline
\end{tabular}

Table 1. The effect of chronic Neuropeptide Y (NPY) administration on blood biochemical indexes in rats. $($ Mean \pm SEM, $n=6)$. Total cholesterol (TC); triglyceride (TG); high density lipoprotein (HDL-C); low density lipoprotein (LDL-C). Ctl: control group, DM: diabetic model group, antiY1R: Y1R receptors antagonist, antiY2R: Y2R receptors antagonist. ${ }^{*} P<0.05$ vs Ctl group, ${ }^{* *} P<0.01$ vs Ctl group, ${ }^{+} P<0.05$ vs DM group, ${ }^{++} P$ $<0.01$ vs DM group.

\section{Measurement of plasma biochemical indices and NPY concentration}

Blood samples of all rats were collected from the heart and then separated for the analysis of total cholesterol (TC), triglycerides (TG), high density lipoprotein (HDL-C), low density lipoprotein (LDL-C) by Enzyme Catalytic Spectrophotometry (Shanghai Rongsheng Biotech Co., Ltd., China) according to instructions of the manufacturer. The concentration of plasma NPY was also measured using a commercial kit (Phoenix Pharmaceutical Company Belmont, CA, USA) [25].

\section{Statistical analysis}

Data were analyzed using one-way ANOVA or Student's $t$-test between two groups and shown as mean \pm SEM. $P<0.05$ was considered as significant difference. Figures were processed by GraphPad Prism 5.0 software (GraphPad Software Inc., San Diego, CA, USA).

\section{Results}

\section{Changes of blood biochemical indices in rats}

The plasma NPY concentration was measured and compared in DM and Ctl rats. Plasma NPY was drastically increased from $6.63 \pm 1.33 \mathrm{ng} / \mathrm{ml}$ in Ctl rats to $15.89 \pm 2.28 \mathrm{ng} / \mathrm{ml}$ in DM rats $(P<0.05$ vs $\mathrm{Ctl}$ group, $\mathrm{n}=6)$. In agreement with previous report[18], our results confirm that DM is correlated with elevated plasma NPY levels. Furthermore, as shown in Table 1, both Y1R antagonist $(P<0.05$ vs DM group, $\mathrm{n}=6)$ and Y2R antagonist $(P<0.01$ vs DM group, $\mathrm{n}=6$ ) almost completely reversed the elevated TC level in DM rats. The increase in TG levels of DM rats was reduced to the level of Ctl rats by Y2R antagonist $(P<0.05$ vs DM group, $\mathrm{n}=6)$, but not by Y1R antagonist $(P>0.05$ vs DM group, $\mathrm{n}=6)$. Though the HDL-C levels demonstrated a slight rise in DM rats compared with Ctl rats, no significant difference was found $(P>0.05, \mathrm{n}=6)$. Nevertheless, elevated HDL-C levels fell sharply in both DM+antiY1R rats $(P<0.01$ vs Ctl group, $P<0.01$ vs DM group, $\mathrm{n}=6)$ and $\mathrm{DM}+$ antiY2R rats $(P<0.05 v s$ Ctl group, $P<0.01$ vs DM group, $\mathrm{n}=6$ ). In addition, the LDL-C level in DM rats increased significantly compared with Ctl rats $(P<0.01, \mathrm{n}=6)$, an effect that was partially suppressed in both DM+antiY1R rats $(P<0.01$ vs Ctl group, $P<0.05$ vs DM group, $\mathrm{n}=6)$ and $\mathrm{DM}+$ antiY2R rats $(P<0.01 v s$. Ctl group, $P<0.05$ vs DM group, $\mathrm{n}=6)$.

\section{Influences of $Y 1 R$ and $Y 2 R$ antagonists on systolic blood pressure and heart rate}

As demonstrated in Fig. 1, the baseline levels of systolic blood pressure (SBP) was significantly decreased from $125.62 \pm 5.81 \mathrm{mmHg}$ in Ctl rats to $78.46 \pm 6.20 \mathrm{mmHg}$ in DM rats $(P<0.01$ vs Ctl group, $\mathrm{n}=6), 69.25 \pm 3.83 \mathrm{mmHg}$ in $\mathrm{DM}+$ antiY1R rats $(P<0.01$ vs Ctl group, $\mathrm{n}=6)$ and $72.13 \pm 3.98 \mathrm{mmHg}$ in $\mathrm{DM}+$ antiY2R rats $(P<0.01$ vs Ctl group, $\mathrm{n}=6$, Fig. 1A). The baseline HR fell from $388.71 \pm 16.59$ beats $/ \mathrm{min}$ in Ctl rats to $306.67 \pm 15.55$ beats/min in DM rats $(P<0.01$ vs Ctl group, $\mathrm{n}=6$, Fig. 1B). This decrement was partially compensated in DM+antiY1R rats $(338.72 \pm 11.46$ beats $/ \mathrm{min}, P<0.05$ vs Ctl group, $\mathrm{n}=6)$ as well as DM+antiY2R rats $(342.00 \pm 13.16$ beats $/ \mathrm{min}, P<0.05$ vs Ctl group, $\mathrm{n}=6$, Fig. 1B). 
A

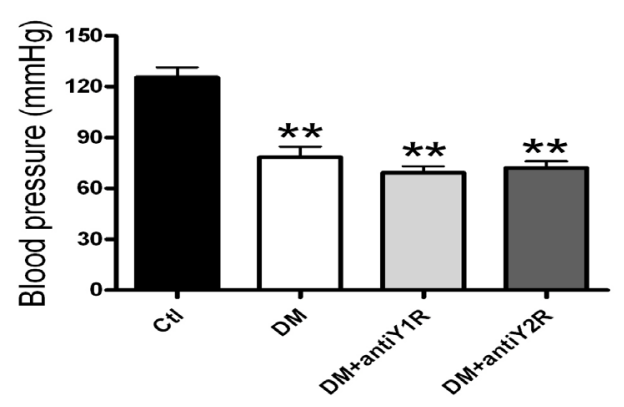

B

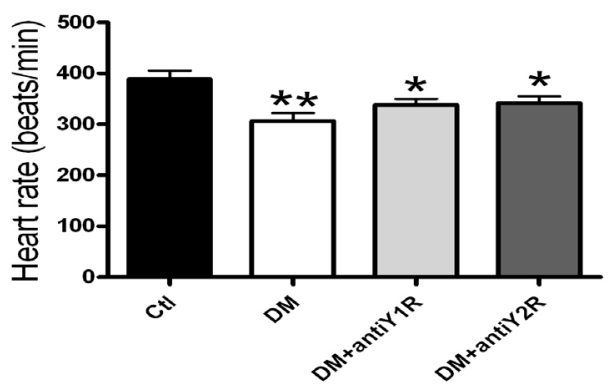

Fig. 1. (A) Baseline systolic blood pressure (SBP) and (B) heart rate (HR) in rats of control group (Ctl), diabetic model group (DM), diabetic model treated with Y1R antagonist group (DM+antiY1R) and diabetic model treated with Y2R antagonist group (DM+antiY2R). ( $\mathrm{n}=6,{ }^{*} P<0.05$ vs. Ctl group; ${ }^{* *} P<0.01$ vs. Ctl group).

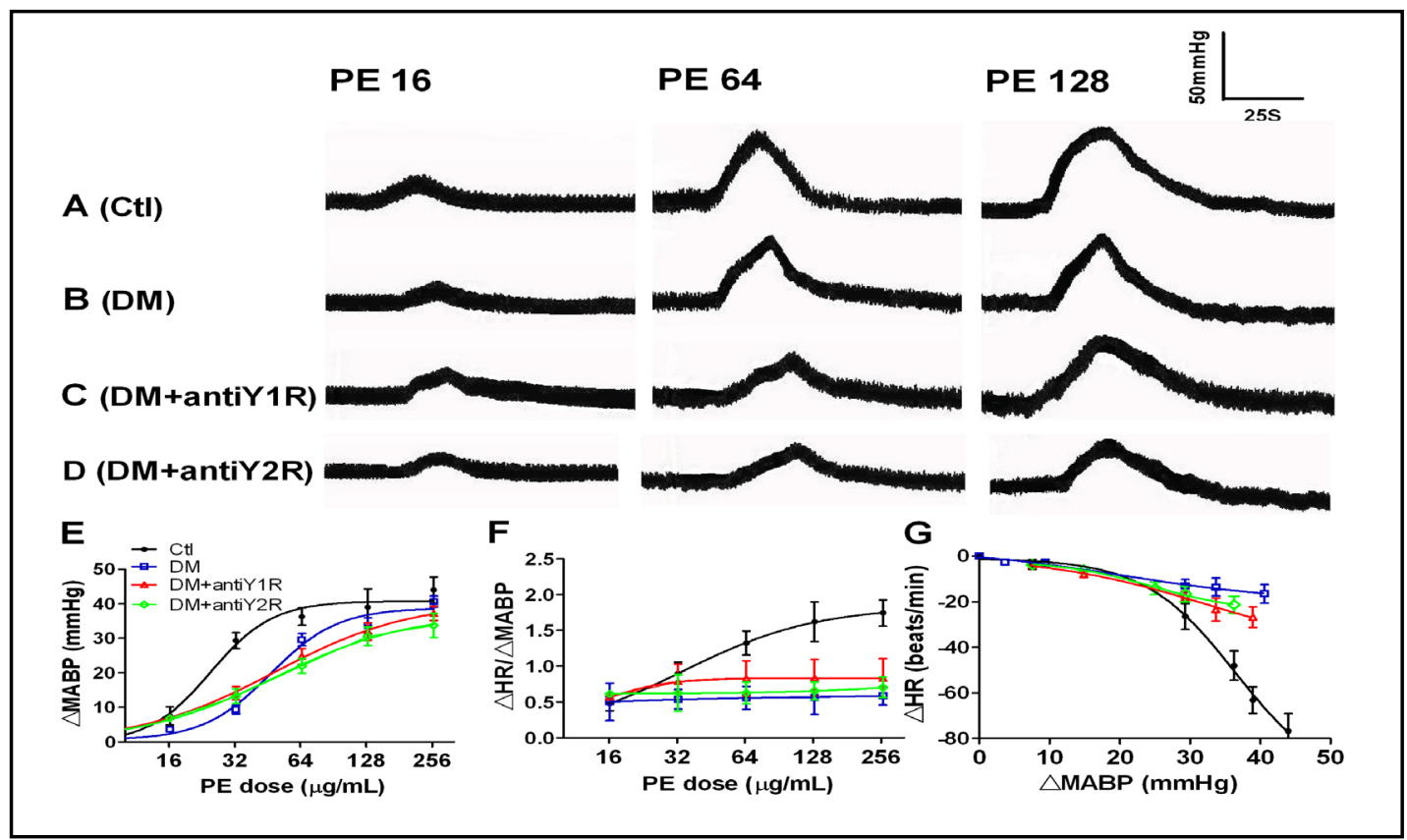

Fig. 2. Baroreceptor reflex control of heart rate in diabetic rats with hyperlipidemia after 4-week treatment with Y1R and Y2R receptors antagonists following phenylephrine (PE) administration. Traces of blood pressure changes induced by PE application at 16, 64, and $128 \mu \mathrm{g} / \mathrm{mL}$ in (A) control rats (Ctl), (B) diabetic model rats with hyperlipidemia (DM), (C) DM rats treated with Y1R receptors antagonist (DM+antiY1R) and (D) DM rats treated with Y2R receptors antagonist (DM+antiY2R). (E) The curve of changes in mean arterial blood pressure $(\triangle \mathrm{MABP})$ against each dose of $\mathrm{PE}$ in the four groups of rats. (F) The curve of $\Delta \mathrm{HR} /$ $\triangle \mathrm{MABP}$ against different doses of PE in the four groups of rats. (G) The curve of $\Delta \mathrm{HR}$ against $\triangle \mathrm{MABP}$ in the four groups of rats. Values are means $(n=6)$ with standard errors represented by vertical bars.

\section{Effects of Y1R and Y2R antagonists on Baroreflex control of heart rate after phenylephrine administration}

As illustrated in Fig. 2, fluctuations of SBP in all groups demonstrated a dose-dependent rise elicited by series doses of phenylephrine (PE). Moreover, the changes of $\triangle \mathrm{MABP}$ over different doses of $\mathrm{PE}$, as displayed in Fig. 2E, showed that these changes were less sensitive in $\mathrm{DM}$ rats than in $\mathrm{Ctl}$ rats (ANOVA, $P<0.05$ vs Ctl group; $\mathrm{n}=6$ ). And neither the $\mathrm{Y} 1 \mathrm{R}$ antagonist (ANOVA; $P>0.05$ vs DM group; $\mathrm{n}=6$; Fig. 2C) nor Y2R antagonist (ANOVA, $P>0.05$ vs DM group $n=6$; Fig.2D) improved the $\triangle$ MABP desensitization induced by DM. For example, at 


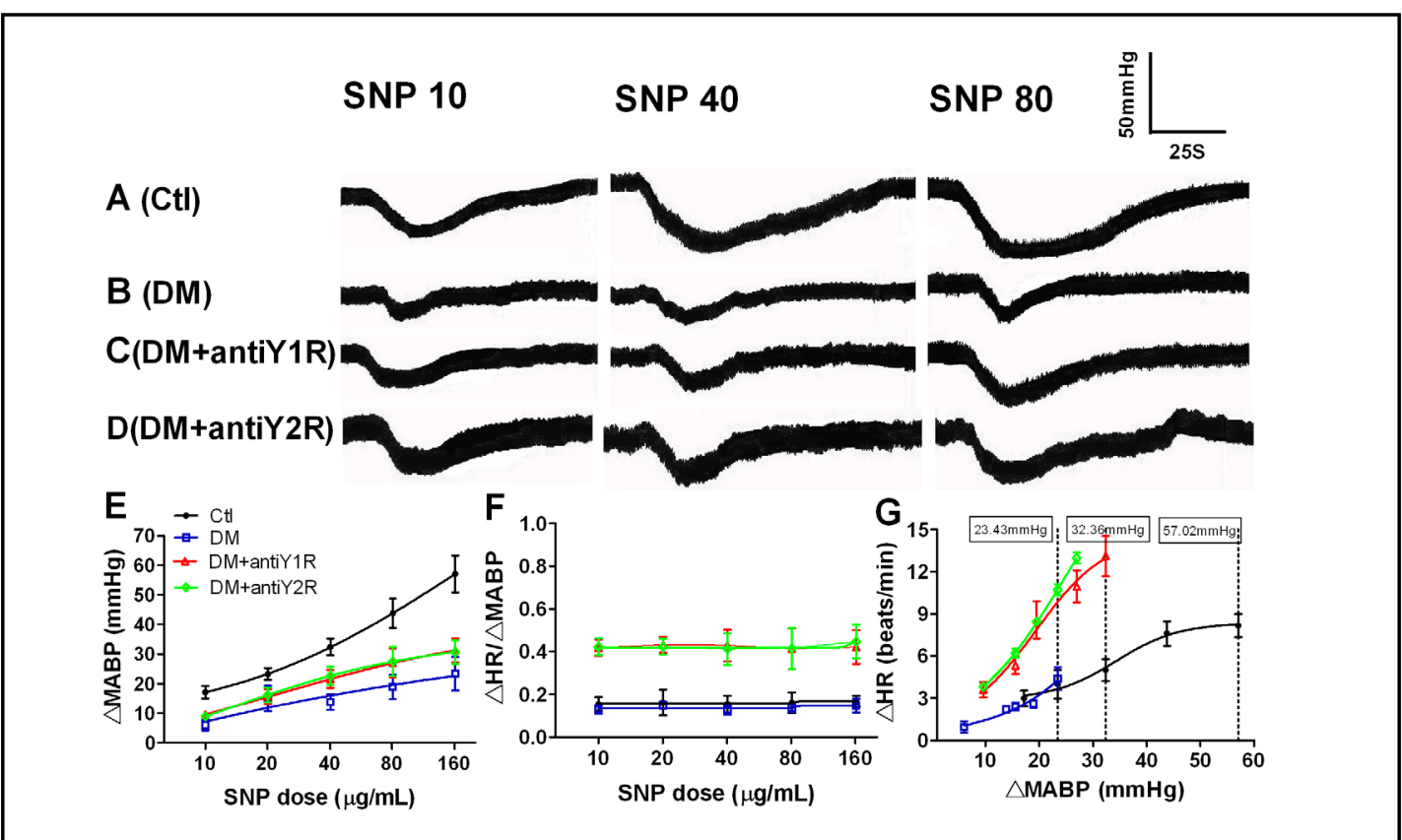

Fig. 3. Baroreceptor reflex control of heart rate in diabetic rats with hyperlipidemia after 4-week treatment with Y1R and Y2R receptors antagonists following sodium nitroprusside (SNP) application. Traces of blood pressure changes induced by SNP application at 10, 40, and $80 \mu \mathrm{g} / \mathrm{mL}$ in (A) control rats (Ctl), (B) diabetic model rats with hyperlipidemia (DM), (C) DM rats treated with Y1R receptors antagonist (DM+antiY1R) and (D) DM rats treated with $\mathrm{Y} 2 \mathrm{R}$ receptors antagonist (DM+antiY2R). (E) The curve of changes in mean arterial blood pressure ( $\triangle \mathrm{MABP}$ ) against each dose of SNP in the four groups of rats. (F) The curve of $\triangle \mathrm{HR} / \triangle \mathrm{MABP}$ against different doses of SNP in the four groups of rats. (G) The curve of $\triangle$ HR against $\triangle$ MABP in the four groups of rats. Values are means $(n=6)$ with standard errors represented by vertical bars.

the dose of $128 \mu \mathrm{g} / \mathrm{ml} \mathrm{PE}$, the value of $\triangle \mathrm{MABP}$ was $38.97 \pm 5.29 \mathrm{mmHg}$ in Ctl rats, $33.67 \pm$ $2.24 \mathrm{mmHg}$ in DM rats, $31.95 \pm 2.50 \mathrm{mmHg}$ in DM+antiY1R rats and $30.55 \pm 2.67 \mathrm{mmHg}$ in $\mathrm{DM}+$ antiY2R, among which no obvious differences were observed.

The curve of $\triangle \mathrm{HR} / \triangle \mathrm{MABP}$ over PE doses was plotted to evaluate BRS. The curve of the DM rats was almost flat which significantly different from the dose-dependent curve of Ctl rats (ANOVA, $P<0.05$ vs Ctl group, $\mathrm{n}=6$, Fig. 2F). Neither the Y1R antagonist (ANOVA, $P>$ 0.05 vs DM group, $\mathrm{n}=6$, Fig. 2F) nor Y2R antagonist (ANOVA, $P>0.05$ vs DM group, $\mathrm{n}=6$, Fig. 2F) treatment had any significant effect on the $\triangle \mathrm{HR} / \triangle \mathrm{MABP}$ after dosed PE treatment when compared with the DM rats. The ratio of $\triangle \mathrm{HR} / \triangle \mathrm{MABP}$ dropped in all other groups when compared with the Ctl group at the dose of $16 \mu \mathrm{g} / \mathrm{ml} \mathrm{PE}$. When challenged by $256 \mu \mathrm{g} /$ $\mathrm{ml} \mathrm{PE}$, the value of $\triangle \mathrm{HR} / \triangle \mathrm{MABP}$ was reduced from $1.74 \pm 0.18$ beats $/ \mathrm{min} / \mathrm{mmHg}$ in Ctl rats to $0.59 \pm 0.13$ beats $/ \mathrm{min} / \mathrm{mmHg}$ in DM rats, similar to the $0.83 \pm 0.27$ beats $/ \mathrm{min} / \mathrm{mmHg}$ in $\mathrm{DM}+$ antiY1 $\mathrm{R}$ rats and the $0.70 \pm 0.15$ beats $/ \mathrm{min} / \mathrm{mmHg}$ in $\mathrm{DM}+$ antiY2 $\mathrm{R}$ groups. .

The maximal $\triangle \mathrm{HR}$ to the maximal $\triangle \mathrm{MABP}$ induced by various doses of PE were monitored as another indicator (Fig. 2G). The maximal $\triangle \mathrm{MABP}$ was repressed to $39.58 \mathrm{mmHg}$ in the DM group, which was fairly consistent with that of DM+antiY1 R (38.97 $\mathrm{mmHg})$ and DM+antiY2R (38.76 mmHg) groups, was reduced compared with Ctl group (43.99 $\mathrm{mmHg}$ ).

Influences of $Y 1 R$ and $Y 2 R$ antagonists on Baroreflex control of heart rate after sodium nitroprusside administration

The amplitude of the decline in ABP induced by doses of SNP grew gradually in all groups as described in Fig. 3A-D. The changes of $\triangle$ MABP elicited by different doses of SNP were blunted in DM rats compared with those in Ctl rats (ANOVA, $P<0.01$ vs Ctl group, 

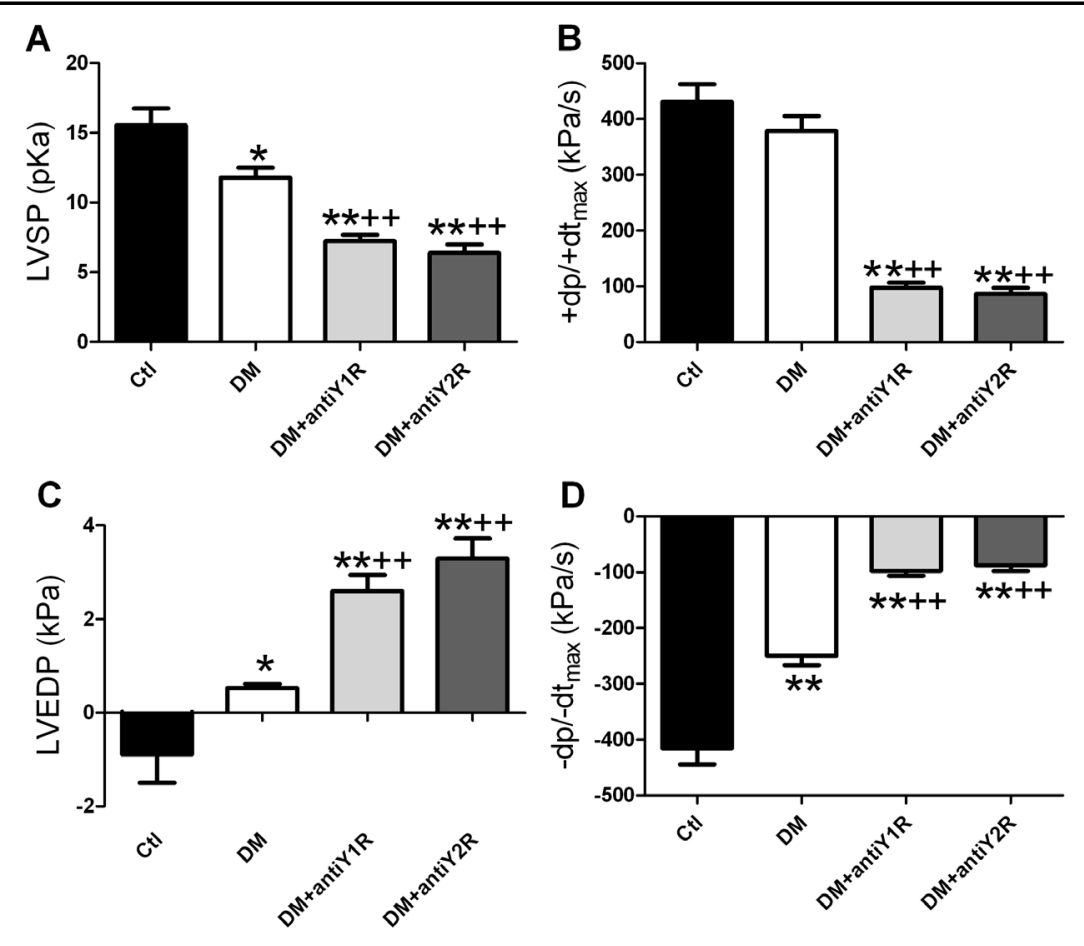

Fig. 4. Quantitative analysis of parameters of cardiac function in control rats (Ctl), diabetic model rats with hyperlipidemia (DM), DM rats treated with Y1R receptors antagonist (DM+antiY1R) and DM rats treated with Y2R receptors antagonist (DM+antiY2R). (A) left ventricular systolic pressure (LVSP); (B) maximum change velocity of left ventricular pressure in the isovolumic contraction period $\left(+\mathrm{dp} / \mathrm{dt}_{\max }\right)$; (C) left ventricular diastolic pressure (LVEDP); (D) maximum change velocity of left ventricular pressure in the isovolumic relaxation period (-dp/dt $\left.{ }_{\max }\right)$. $\left(\mathrm{n}=6,{ }^{*} P<0.05\right.$ vs Ctl group; ${ }^{* *} P<0.01$ vs Ctl group; ${ }^{++} P<0.01$ vs DM group)

$\mathrm{n}=6$; Fig. 3B), and the $\triangle \mathrm{MABP}$ values in DM+antiY1R (ANOVA, $P<0.05$ vs Ctl group; $P<$ 0.05 vs DM group, $\mathrm{n}=6$, Fig. $3 \mathrm{C}$ ) and DM+antiY2R (ANOVA, $P<0.05$ vs Ctl group; $P<0.05$ $v s$ DM group, $\mathrm{n}=6$, Fig. 3D) groups were partly ameliorated compared with the DM group. As indicated in Fig. $3 \mathrm{~F}$, the flat curves of $\triangle \mathrm{HR} / \triangle \mathrm{MABP}$ against SNP doses of the Ctl and DM group were almost equivalent (ANOVA, $P>0.05$ vs Ctl group, $\mathrm{n}=6$ ) with the linear curves of $\mathrm{DM}+$ antiY1R (ANOVA, $P<0.01$ vs Ctl group; $P<0.01$ vs DM group, $\mathrm{n}=6$ ) and $\mathrm{DM}+$ antiY2R group (ANOVA, $P<0.01$ vs Ctl group; $P<0.01$ vs DM group, $\mathrm{n}=6$ ) above. As for the maximal $\triangle \mathrm{HR}$ to the maximal $\triangle \mathrm{MABP}$ induced by various doses of SNP, the maximal $\triangle \mathrm{MABP}$ decreased from $57.02 \mathrm{mmHg}$ in the Ctl rats to $23.43 \mathrm{mmHg}$ in the DM rats, which was partially reversed to $32.36 \mathrm{mmHg}$ in both the DM+antiY1R and DM+antiY2R rats (Fig. 3G).

\section{Alterations of heart function after $Y 1 R$ and $Y 2 R$ antagonists treatment}

Fig. 4 indicated that the damage of cardiac function of DM rats was further deteriorated by the blockage of Y1R or Y2R. To evaluate cardiac systolic function, LVSP and $+\mathrm{d} p / \mathrm{d} t_{\max }$ were studied. Compared with Ctl rats (LVSP: $15.53 \pm 1.22 \mathrm{kPa} ;+\mathrm{d} p / \mathrm{d} t_{\text {max }}: 431.00 \pm 31.09$ $\mathrm{kPa} / \mathrm{s}), \mathrm{DM}$ rats displayed significantly decreased LSVP $(11.79 \pm 0.70 \mathrm{kPa}, P<0.05$ vs Ctl group, $\mathrm{n}=6$ ) and negligible $+\mathrm{d} p / \mathrm{d} t_{\max }$ reduction $(378.33 \pm 26.75 \mathrm{kPa} / \mathrm{s}, P>0.05$ vs Ctl group, $\mathrm{n}=6$ ). Notably, Y1R antagonist worsened the impaired systolic function with the value of LVSP falling to $7.23 \pm 0.45 \mathrm{kPa}(P<0.01$ vs Ctl group, $P<0.01$ vs DM group, $\mathrm{n}=6)$ and $+\mathrm{d} p /$ $\mathrm{d} t_{\text {max }}$ decreasing to $97.75 \pm 9.27 \mathrm{kPa} / \mathrm{s}(P<0.01$ vs Ctl group, $P<0.01$ vs DM group, $\mathrm{n}=$ 6). Similar to the DM+antiY1R group observations, the DM+antiY2R group LVSP decreased to $6.39 \pm 0.60 \mathrm{kPa}(P<0.01$ vs Ctl group, $P<0.01$ vs DM group, $\mathrm{n}=6)$ and $+\mathrm{d} p / \mathrm{d} t_{\max }$ was repressed to $86.63 \pm 10.56 \mathrm{kPa} / \mathrm{s}(P<0.01$ vs Ctl group, $P<0.01$ vs DM group, $\mathrm{n}=6$, Fig. 4A-B). 
With regards to diastolic function, LVEDP was impaired in DM rats $(0.53 \pm 0.09 \mathrm{kPa}, P<0.05$ vs Ctl group, $\mathrm{n}=6)$ compared with Ctl rats $(-0.90 \pm 0.60 \mathrm{kPa})$. Both Y1R antagonist $(2.60 \pm$ $0.34 \mathrm{kPa}, P<0.01$ vs Ctl group, $P<0.01$ vs DM group, $\mathrm{n}=6)$ and $\mathrm{Y} 2 \mathrm{R}$ antagonist $(3.29 \pm 0.43$ $\mathrm{kPa}, P<0.01$ vs Ctl group, $P<0.01$ vs DM group, $\mathrm{n}=6$, Fig. $4 \mathrm{C}$ ) amplified the damage. The influences on $-\mathrm{d} p / \mathrm{d} t_{\text {max }}$ were similar to the tendency of LVEDP. The value of $-\mathrm{d} p / \mathrm{d} t_{\text {max }}$ was significantly increased from $-415.71 \pm 28.72 \mathrm{kPa} / \mathrm{s}$ in Ctl rats to $-249.71 \pm 16.96 \mathrm{kPa} / \mathrm{s}$ in DM rats $(P<0.01$ vs Ctl group, $\mathrm{n}=6)$, and this was enlarged in $\mathrm{DM}+$ antiY1R rats $(-97.90 \pm 8.16$ $\mathrm{kPa} / \mathrm{s}, P<0.01$ vs Ctl group, $P<0.01$ vs DM group, $\mathrm{n}=6)$ and $\mathrm{DM}+$ antiY2R rats $(-87.47 \pm 10.62$ $\mathrm{kPa} / \mathrm{s}, P<0.01$ vs Ctl group, $P<0.01$ vs DM group, $\mathrm{n}=6$, Fig. 4D).

\section{Discussion}

In the present study, we initially found that the blockage of peripheral Y1R and Y2R in BRS showed no effects on BRS, baseline SBP or HR, but that inhibiting these receptors further suppressed the already impaired cardiac function in DM rats with hyperlipidemia. This discovery provides increased insights on the role of Y1R and Y2R in cardiovascular autonomic neuropathy associated with DM.

We established a DM model with hyperlipidemia using STZ induction accompanied by oral administration of fat emulsion, and showed that DM lead to increased plasma NPY. Previous studies have reported that raised plasma NPY is highly associated with more severe condition and prognosis under multiple pathological states $[13,14]$. Based on the results, we hypothesized that the elevated plasma NPY might negatively influences BRS and cardiac function via activation of Y1R and Y2R in DM. With the consideration that they could not cross the blood brain barrier, we employed BIBP 3226 and BIIE 0246 as Y1R and Y2R antagonists respectively [26, 27]. Based on the essential role of lipid metabolism in cardiovascular dysfunction of DM, we measured the plasma lipid concentration and found that BIBP 3226 and BIIE 0246 rescued the aberrant lipid level. Previous studies suggest that peripheral Y1R participates in the regulation of energy homeostasis mainly via fatty acid oxidation [28], while Y2R located in abdominal adipocytes takes part in the process of stress-induced obesity on peripheral fat tissue [29]. These properties of Y1R/Y2R implied that they might also similarly influence the fat metabolism and lipid modulation in a DM model. However, the pathways by which Y1R/Y2R regulate the abnormal lipid levels in DM rats with hyperlipidemia remain unclear and deserve further study.

We recorded the baseline SBP and HR in the four groups of rats and our results showed that BIBP 3226 and BIIE 0246 did not exert any obvious effects on DM-induced hypotension and bradycardia. Further more, blockage of Y1R did not impact baseline SBP and HR, which was consistent with a previous study on Y1R knockout mice [30]. It was reported that Y1R deleted mice showed normal baseline SBP and HR, even though the vasoconstrictor action of both NPY and noradrenaline-induced BP modulation were blocked in these mice. Despite the increased baseline HR of Y2R deficient mice, the baseline SBP was also reported unaffected $[31,32]$. It is worth noting that some of these animal models were involved central Y1R/ $\mathrm{Y} 2 \mathrm{R}$, and revealed an integrated role of Y1R/Y2R in balancing baseline SBP and HR. Although central Y1R/Y2R might play an important role, the majority of Y1R/Y2R is distributed in the periphery, where they are also indispensable in modulating baseline SBP and HR. It has been well documented that Y1R/Y2R influence the SBP through several pathways. Firstly, NPY could directly act on Y1R in vascular smooth muscle to contract blood vessels postjunctionally $[33,34]$. Secondly, NPY could fuel the effects of numerous mediators on vascular tone via Y1R [35]. Thirdly, NPY could act on Y2R and inhibit the release of NE by a presynaptic mechanism [36]. Consequently, the constant baseline SBP and HR after BIBP 3226 and BIIE 0246 administration should be multi-factorial outcomes as discussed above. In spite of the direct effect of Y2R on cardiac contraction [37], the baseline HR was more likely to be indirectly regulated by other factors, including those associated with the baseline SBP and finally achieved an unvaried state. 
We tested our hypothesis that elevated plasma NPY impairs BRS by acting on peripheral Y1R and Y2R in DM rats. The data indicated that DM rats with hyperlipidemia suffered severely from impaired BRS, particularly demonstrated by the responses to PE challenges. Consistent with our previous study [20], the curve of $\triangle \mathrm{HR} / \triangle \mathrm{MABP}$ at serial doses of SNP in the DM group was flat and showed no significant difference with that of the Ctl group. This might be partially attributed to the inhibition of baroreflex function induced by the hypotensive and anesthetic state [20,38,39]. Contrary to our hypothesis, both BIBP 3226 and BIIE 0246 administration showed no obvious influence to the DM-induced impairment of BRS when challenged by serial doses of PE or SNP. This is consistent with the previous findings that bolus intravenous infusion of GR23118, another specific Y1R antagonist, had no effect on cardiac baroreceptor-HR reflex in conscious rabbits [40]. Although the treatment durations between the two studies were varied ( 4 weeks $v s$ bolus), both of their antagonists were systemically administrated, antagonized at similar sites, and identically affected on the BRS. Numerous peripheral pathological changes at the site of the baroreceptors (such as damaged cardiac vagal function, left ventricular hypertrophy, endothelial dysfunction, decreased distensibility and increased intima-media thickness) combine to achieve the impaired BRS in DM [3].

As BIBP 3226 and BIIE 0246 showed no effect on DM-elicited BRS impairments, we then tried to determine whether they have any effect on diabetic cardiac function. Unexpectedly, DM-induced impairment of systolic function in parallel with diastolic function was enormously deteriorated by both BIBP 3226 and BIIE 0246, which revealed the benefits of activating Y1R and Y2R in preserving DM-induced cardiac function. It is well established that diabetic cardiomyopathy involves not only sensory and autonomic innervation but also the contractile cardiomyocytes. BIBP 3226 and BIIE 0246 elicited deterioration of the compromised cardiac function in DM rats through several possible pathways. In a direct fashion, NPY could constrict coronary [33], potentiate NE-induced constriction [41] and weaken the NE-induced relaxation [42]. Indirectly, NPY could positively or negatively alter preload by venous constriction and induce a secondary histamine release respectively [35]. Meanwhile NPY could alter afterload through vasoconstrictor activities via itself or other vasoconstrictors potentiated by NPY [35]. In addition, activated Y1R and Y2R in the endocardial endothelial cells (EECs) were reported to lead to increased steady-state level of intracellular free $\mathrm{Ca}^{2+}$ in EECs and therefore contribute to the performance of cardiac function [43]. The BIBP 3226 and BIIE 0246 induced degeneration of cardiac function in our study lend credit to the proposal that DM-elicited plasma NPY positively preserved the damaged cardiac function via activating peripheral Y1R and Y2R. The exact mechanisms by which the activated Y1R and Y2R serve as positive protectors of impaired cardiac function in DM state are still to be elucidated.

In conclusion, the blockade of peripheral Y1R and Y2R by specific antagonist (BIBP 3226 and BIIE 0246) had no effect on BRS, baseline SBP and HR in STZ-induced diabetic rats with hyperlipidemia. However, antagonism of peripheral Y1R and Y2R significantly impaired cardiac function in DM, which suggests a protective role of elevated plasma NPY in the maintenance of DM elicited cardiac function.

\section{Acknowledgements}

This work was supported by Natural Science Foundation of China (81271207, 81070882), Outstanding Youth Foundation of Heilongjiang Province (JC200904). Creative Research Groups of the National Natural Science Foundation of China (81121003). 


\section{Cellular Physiology $\quad$ Cell Physiol Biochem 2013;31:421-431

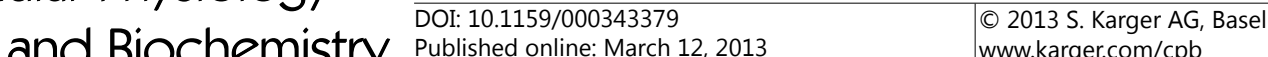 \\ Liu/Wang/Xie/Sun/Chen/Ye/Cai/Yang/Ai: Role of Y1R/Y2R in BRS of Diabetic Rats}

\section{References}

1 Rubler S, Dlugash J, Yuceoglu YZ, Kumral T, Branwood AW, Grishman A: New type of cardiomyopathy associated with diabetic glomerulosclerosis. Am J Cardiol 1972;30:595-602.

- Schwartz PJ, Vanoli E, Crotti L, Spazzolini C, Ferrandi C, Goosen A, Hedley P, Heradien M, Bacchini S, Turco A, La Rovere MT, Bartoli A, George AL Jr, Brink PA: Neural control of heart rate is an arrhythmia risk modifier in long qt syndrome. J Am Coll Cardiol 2008;51:920-929.

-3 Lefrandt JD, Smit AJ, Zeebregts CJ, Gans RO, Hoogenberg KH: Autonomic dysfunction in diabetes: A consequence of cardiovascular damage. Curr Diabetes Rev 2010;6:348-358.

4 Ziegler D, Gries FA, Spuler M, Lessmann F: The epidemiology of diabetic neuropathy. Diabetic cardiovascular autonomic neuropathy multicenter study group. J Diabetes Complications 1992;6:49-57.

-5 La Rovere MT, Pinna GD, Hohnloser SH, Marcus FI, Mortara A, Nohara R, Bigger JT Jr, Camm AJ, Schwartz PJ: Baroreflex sensitivity and heart rate variability in the identification of patients at risk for life-threatening arrhythmias: Implications for clinical trials. Circulation 2001;103:2072-2077.

-6 Stein KM: Noninvasive risk stratification for sudden death: Signal-averaged electrocardiography, nonsustained ventricular tachycardia, heart rate variability, baroreflex sensitivity, and qrs duration. Prog Cardiovasc Dis 2008;51:106-117.

7 Yan B, Li L, Harden SW, Epstein PN, Wurster RD, Cheng ZJ: Diabetes induces neural degeneration in nucleus ambiguus (na) and attenuates heart rate control in ove26 mice. Exp Neurol 2009;220:34-43.

8 Bjorntorp P, Rosmond R: Neuroendocrine abnormalities in visceral obesity. Int J Obes Relat Metab Disord 2000;24 Suppl 2:S80-85.

-9 Walker P, Grouzmann E, Burnier M, Waeber B: The role of neuropeptide y in cardiovascular regulation. Trends Pharmacol Sci 1991;12:111-115.

-10 Testa A, Mallamaci F, Macri R, Pisano A, Spoto B, Malatino LS, Stancanelli B, Tripepi G, Benedetto FA, Zoccali C: Neuropeptide y receptor y2 gene polymorphism interacts with plasma neuropeptide y levels in predicting left ventricular hypertrophy in dialysis patients. J Hypertens 2010;28:1745-1751.

11 Westfall TC: Neuropeptide y and sympathetic control of vascular tone in hypertension. EXS 2006;95:89103.

12 Ullman B, Franco-Cereceda A, Hulting J, Lundberg JM, Sollevi A: Elevation of plasma neuropeptide y-like immunoreactivity and noradrenaline during myocardial ischaemia in man. J Intern Med 1990;228:583589.

13 Hulting J, Sollevi A, Ullman B, Franco-Cereceda A, Lundberg JM: Plasma neuropeptide y on admission to a coronary care unit: Raised levels in patients with left heart failure. Cardiovasc Res 1990;24:102-108.

-14 Ullman B, Lindvall K, Lundberg JM, Sigurdsson A, Swedberg K: Response of plasma neuropeptide y and noradrenaline to dynamic exercise and ramipril treatment in patients with congestive heart failure. Clin Physiol 1994;14:123-134.

15 Cohen M, Parry G, Adams PC, Xiong J, Chamberlain D, Wieczorek I, Fox KA, Kronmal R, Fuster V: Prospective evaluation of a prostacyclin-sparing aspirin formulation and heparin/warfarin in aspirin users with unstable angina or non-q wave myocardial infarction at rest. The antithrombotic therapy in acute coronary syndromes research group. Eur Heart J 1994;15:1196-1203.

16 Xie F, Zhang R, Yang C, Xu Y, Wang N, Sun L, Liu J, Wei R, Ai J: Long-term neuropeptide y administration in the periphery induces abnormal baroreflex sensitivity and obesity in rats. Cell Physiol Biochem 2012;29:111-120.

17 Xie F, Sun L, Su X, Wang Y, Liu J, Zhang R, Wang N, Zhao J, Ban T, Niu H, Ai J: Neuropeptide y reverses chronic stress-induced baroreflex hypersensitivity in rats. Cell Physiol Biochem 2012;29:463-474.

-18 Ilhan A, Rasul S, Dimitrov A, Handisurya A, Gartner W, Baumgartner-Parzer S, Wagner L, Kautzky-Willer A, Base W: Plasma neuropeptide y levels differ in distinct diabetic conditions. Neuropeptides 2010;44:485489.

19 Uddman R, Moller S, Nilsson T, Nystrom S, Ekstrand J, Edvinsson L: Neuropeptide y y1 and neuropeptide y y2 receptors in human cardiovascular tissues. Peptides 2002;23:927-934.

20 Ai J, Wang LH, Zhang R, Qiao GF, Wang N, Sun LH, Lu GY, Sun C, Yang BF: Protective effect of the daming capsule on impaired baroreflexes in stz-induced diabetic rats with hyperlipoidemia. BMC Complement Altern Med 2010;10:80. 


\section{Cellular Physiology $\quad$ Cell Physiol Biochem 2013;31:421-431 and Biochemistry \\ Liu/Wang/Xie/Sun/Chen/Ye/Cai/Yang/Ai: Role of Y1R/Y2R in BRS of Diabetic Rats}

21 Ai J, Yan X, Zhao L, Lu Y, Liang F, Cai B, Li G, Yang B: The protective effect of daming capsule on heart function in streptozocin-induced diabetic rats with hyperlipidemia. Biol Pharm Bull 2009;32:1354-1358.

22 Ai J, Liang F, Zhou H, Zhao J, Wang N, Zhu S, Yang B: Mechanism of impaired baroreflex sensitivity in wistar rats fed a high-fat and -carbohydrate diet. Br J Nutr 2010;104:291-297.

23 Lin M, Liu R, Gozal D, Wead WB, Chapleau MW, Wurster R, Cheng ZJ: Chronic intermittent hypoxia impairs baroreflex control of heart rate but enhances heart rate responses to vagal efferent stimulation in anesthetized mice. Am J Physiol Heart Circ Physiol 2007;293:H997-1006.

24 Patel KP, Zhang PL: Baroreflex function in streptozotocin (stz) induced diabetic rats. Diabetes Res Clin Pract 1995;27:1-9.

25 Morris M, Kapoor V, Chalmers J: Plasma neuropeptide y concentration is increased after hemorrhage in conscious rats: Relative contributions of sympathetic nerves and the adrenal medulla. J Cardiovasc Pharmacol 1987;9:541-545.

26 Brothers SP, Saldanha SA, Spicer TP, Cameron M, Mercer BA, Chase P, McDonald P, Wahlestedt C, Hodder PS: Selective and brain penetrant neuropeptide y y2 receptor antagonists discovered by whole-cell highthroughput screening. Mol Pharmacol 2010;77:46-57.

27 Leupen SM, Besecke LM, Levine JE: Neuropeptide y y1-receptor stimulation is required for physiological amplification of preovulatory luteinizing hormone surges. Endocrinology 1997;138:2735-2739.

28 Zhang L, Macia L, Turner N, Enriquez RF, Riepler SJ, Nguyen AD, Lin S, Lee NJ, Shi YC, Yulyaningsih E, Slack K, Baldock PA, Herzog H, Sainsbury A: Peripheral neuropeptide y y1 receptors regulate lipid oxidation and fat accretion. Int J Obes (Lond) 2009;34:357-373.

-29 Kuo LE, Kitlinska JB, Tilan JU, Li L, Baker SB, Johnson MD, Lee EW, Burnett MS, Fricke ST, Kvetnansky R, Herzog H, Zukowska Z: Neuropeptide y acts directly in the periphery on fat tissue and mediates stressinduced obesity and metabolic syndrome. Nat Med 2007;13:803-811.

-30 Pedrazzini T, Seydoux J, Kunstner P, Aubert JF, Grouzmann E, Beermann F, Brunner HR: Cardiovascular response, feeding behavior and locomotor activity in mice lacking the npy y1 receptor. Nat Med 1998;4:722-726.

-31 Naveilhan P, Hassani H, Canals JM, Ekstrand AJ, Larefalk A, Chhajlani V, Arenas E, Gedda K, Svensson L, Thoren P, Ernfors P: Normal feeding behavior, body weight and leptin response require the neuropeptide y y2 receptor. Nat Med 1999;5:1188-1193.

-32 Smith-White MA, Herzog H, Potter EK: Role of neuropeptide y y(2) receptors in modulation of cardiac parasympathetic neurotransmission. Regul Pept 2002;103:105-111.

-33 Clarke JG, Davies GJ, Kerwin R, Hackett D, Larkin S, Dawbarn D, Lee Y, Bloom SR, Yacoub M, Maseri A: Coronary artery infusion of neuropeptide y in patients with angina pectoris. Lancet 1987;1:1057-1059.

-34 Aizawa Y, Satoh M, Aizawa M, Funazaki T, Niwano S, Miyajima S, Shibata A: Potency and receptors involved in coronary vasoconstriction caused by neuropeptide y(npy). Jpn Heart J 1987;28:891-898.

-35 Edvinsson L, Ekblad E, Hakanson R, Wahlestedt C: Neuropeptide y potentiates the effect of various vasoconstrictor agents on rabbit blood vessels. Br J Pharmacol 1984;83:519-525.

-36 Serone AP, Wright CE, Angus JA: Heterogeneity of prejunctional npy receptor-mediated inhibition of cardiac neurotransmission. Br J Pharmacol 1999;127:99-108.

-37 McDermott BJ, Bell D: Npy and cardiac diseases. Curr Top Med Chem 2007;7:1692-1703.

- 38 Shimokawa A, Kunitake T, Takasaki M, Kannan H: Differential effects of anesthetics on sympathetic nerve activity and arterial baroreceptor reflex in chronically instrumented rats. J Auton Nerv Syst 1998;72:46-54.

-39 Van Leeuwen AF, Evans RG, Ludbrook J: Effects of halothane, ketamine, propofol and alfentanil anaesthesia on circulatory control in rabbits. Clin Exp Pharmacol Physiol 1990;17:781-798.

40 Serone AP, Wright CE, Angus JA: Role of npy y1 receptors in cardiovascular control in the conscious rabbit. J Cardiovasc Pharmacol 2000;35:315-321.

41 Prieto D, Benedito S, Simonsen U, Nyborg NC: Regional heterogeneity in the contractile and potentiating effects of neuropeptide y in rat isolated coronary arteries: Modulatory action of the endothelium. Br J Pharmacol 1991;102:754-758.

42 Corr L, Burnstock G, Poole-Wilson P: Effects of age and hyperlipidemia on rabbit coronary responses to neuropeptide y and the interaction with norepinephrine. Peptides 1993;14:359-364.

43 Abdel-Samad D, Jacques D, Perreault C, Provost C: Npy regulates human endocardial endothelial cell function. Peptides 2007;28:281-287. 